\title{
Natural copper stable isotopes in the detection of Alzheimer's Disease
}

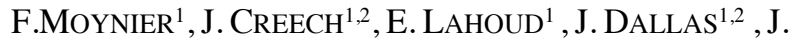

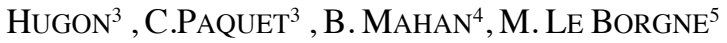 \\ ${ }^{1}$ Institut de Physique du Globe de Paris, Université de Paris, \\ CNRS, 1 rue Jussieu 75005 Paris, moynier@ipgp.fr \\ ${ }^{2}$ Department of Earth and Planetary Sciences, Macquarie \\ Unviersity, Sydney, New South Wales 2109, Australia $^{3}$ \\ ${ }^{3}$ Centre de Neurologie Cognitive, Hopital Lariboisière APHP \\ Université de Paris, France \\ ${ }^{4}$ Department of Earth and Environmental Sciences, James \\ Cook University, Townsville, Queensland 4814, Australia \\ ${ }^{5}$ Unité 1148 (INSERM), Hôpital Xavier Bichat, Université de \\ Paris.
}

Alzheimer's disease (AD) is a leading cause of loss of autonomy in high-income countries, and afflicts one out of ten people 65 or older worldwide. One of the major physiological features of $\mathrm{AD}$ is the formation of senile plaques associated with extracellular deposition of metal-rich (e.g. $\mathrm{Cu}, \mathrm{Zn}$ ) amyloid $\beta(\mathrm{A} \beta)$ fibrils. The natural stable isotopic composition of metals such as $\mathrm{Zn}, \mathrm{Cu}$ and $\mathrm{Fe}$ vary between bodily organs and show very limited natural variations. They are therefore powerful tracers of metal inbalance in the body. In particular, blood is isotopically distinct in $\mathrm{Zn}, \mathrm{Cu}$ and $\mathrm{Fe}$ from the brain. The speciation (bonding) of metals in $A \beta$ fibrils is different to that in the healthy brain, and this can modify the isotopic distribution of metals between the brain and body fluids. Here we will present serum and brain $\mathrm{Cu}$ isotopic compositions of (i) APPswe/PSEN1dE9 transgenic mice (a model of AD), (ii) wild-type (WT) controls for 3, 6, 9 and 12-month-old mice (data published), (iii) human brain tissues including both AD and healthy patients (data acquired but unpublished), and (iv) $\mathrm{AD}$-afflicted and healthy human cerebropinal fluids (work in progress). We find that $\mathrm{AD}$-afflicted brains of mice and humans are isotopically lighter for $\mathrm{Cu}$ than brains of healthy mice and patients. We suggest that this reflects an increase of $\mathrm{Cu}(\mathrm{I})$ associated with the formation of $\mathrm{A} \beta$ fibrils. In the mice, the $\mathrm{Cu}$ isotopic composition of the brains and serum were correlated, implying copper transport between these two reservoirs, in particular a transfer of $\mathrm{Cu}(\mathrm{I})$ from the brain to the serum. These data suggest that the $\mathrm{Cu}$ stable isotopic composition of body fluid(s) may have the potential to be used as detection tools for the formation of $A \beta$ fibrils in the brain. 